\title{
¿Por qué partir de la Investigación Acción Participativa para transformar la educación?
}

Un análisis desde la propuesta de Fe y Alegría Ecuador

María Noelle Acosta V. ${ }^{\mathbf{8}}$

manoelleacosta@hotmail.com

Emely Benavides V. ${ }^{9}$

emely.benavides@feyalegria.org.ec

Artículo recibido en mayo de 2018 y aceptado en junio2018

\section{Resumen}

Este artículo plantea la importancia de la Investigación Acción Participativa (IAP) en la esfera educativa y su convergencia con la Educación Popular, en tanto ambas persiguen la transformación social a partir del desarrollo de una conciencia crítica. Propone una forma de concebir la investigación comprometida con el cambio social y, en consecuencia, quienes asumen el reto de adoptarla están llamados a la acción. Es así como la IAP plantea constantemente el desafío de desmitificar el supuesto de que no es posible alterar la educación formal tal como ha sido concebida. A partir del proceso emprendido desde el Centro de Formación e Investigación Fe y Alegría Ecuador (CFI) buscamos dar luces sobre un posible camino para provocar en las y los educadores el deseo de asumirse como agentes llamados a reinventar creativa y críticamente el proceso educativo.

Palabras clave: Investigación-Acción-Participativa, transformación, educadores/as investigadores/as, pensamiento crítico, construcción colectiva.

8 Máster en Cooperación al Desarrollo con especialidad en Desarrollo Local por la Universidad de Valencia. Licenciada en Comunicación por la Universidad de Los Hemisferios. Investigadora asociada al Centro de Formación e Investigación Fe y Alegría Ecuador.

9 Coordinadora de Investigación del Centro de Formación e Investigación de Fe y Alegría Ecuador. Lic. en Sociología con mención en Desarrollo por la Pontificia Universidad Católica del Ecuador. 


\section{Abstrac}

This article focuses on the importance of Participatory Action Research (PAR) in the educational sphere and its convergence with Popular Education, while both pursue social transformation from the development of a critical consciousness. It proposes a way of conceiving research committed to social change and, consequently, those who assume the challenge of adopting it are called to action. This is how PAR constantly brings the challenge of demystifying the assumption that it is not possible to upset formal education as it has been conceived. From the process undertaken by the Training and Research Center (TRC) of Fe y Alegria Ecuador, we seek to give an insight on a possible way to provoke in educators their wish to be assumed as committed agents to reinvent creatively and critically the educational process.

Key words: Participatory Action Research, transformation, educators, researchers, critical thinking, collective construction.

\section{Introducción}

Es ampliamente reconocido que la escuela formal está llamada a ser deconstruida. Si admitimos la condición estática y alienante que puede llegar a asumir el proceso educativo desde las aulas, ¿por qué entonces quienes promulgamos las necesidades de cambio no hemos generado los mecanismos suficientes para que lo expresado en los discursos se materialice en la práctica? Se nos plantea el desafío de articularnos educadores/as, estudiantes, académicos y académicas, y todos quienes visualizan y están comprometidos con la posibilidad de un cambio.

Estamos inmersos en rutinas que resultan ser distractoras de la realidad y que refuerzan la necesidad de mantener el statu quo. La institución educativa dominante ha condicionado nuestra manera de percibir la educación $y$, en esta medida, ha puesto límites a nuestra imaginación crítica creativa; nos ha atribuido a los educadores y educadoras el papel de reproductores (as) o ejecutores (as) de tareas, de currículo o de políticas; cerrando la posibilidad de ver alternativas reales capaces de alterar de manera sustancial lo que conocemos y hacemos. Esta incapacidad de advertir y asumir nuestro rol como agentes capaces 
de transformar, que es reafirmada constantemente por el sistema, debe ser desmitificada a través de la acción.

La Investigación Acción Participativa (IAP) aparece como una alternativa de construcción de conciencia que supone la articulación de diversos actores con el fin de emprender acciones que desestabilicen el sistema educativo desde sus cimientos y proponga, a partir de la práctica, nuevos caminos para comprender y hacer educación.

Este artículo busca profundizar en el rol de la IAP dentro de la esfera educativa y su particular correlación con la Educación Popular. Asimismo, pretende visibilizar la importancia de generar procesos en los que las y los educadores se asuman también como investigadores/ as. A partir del breve recorrido que desde el Centro de Formación e Investigación Fe y Alegría Ecuador (CFI) ha emprendido, queremos dar luces de alternativas para poner en marcha la IAP en centros educativos.

\section{La convergencia entre Educación Popular e Investigación Acción Participativa}

¿Por qué partir de la Investigación Acción Participativa (IAP) para transformar la educación? Este cuestionamiento nos remite en primera instancia al por qué de la IAP, de una investigación sacada de las barreras que la encasillan en la academia para llevarla a diversos contextos, a la multiplicidad de realidades que conforman las sociedades. La IAP como su nombre refiere, parte de investigar una situación conflictiva de manera participativa para transformarla a través de acciones concretas. Quizás solo el hacer alusión a lo que este tipo de investigación nos llama, esclarece por qué debe ser situada en realidades cotidianas; también en la academia ¿por qué no? pero no solamente en ella, pues pretender transformar una realidad social, supone la toma de conciencia y el empoderamiento de quienes están inmersas en ella. En definitiva, lo que se plantea es la posibilidad (de todas las personas) de indagar en las problemáticas que las aquejan, cuestionar, problematizar su contexto, escucharse y apoyarse mutuamente para incidir en ellas, y ese incidir, esa toma de acción, implica un compromiso para quienes se embarcan en este propósito. 
La IAP se nos presenta entonces como una metodología que posibilita crear círculos virtuosos de "reflexión-diálogo-acciónaprendizaje" (Durston \& Miranda, 2002, pág. 9). Círculos que se retroalimentan constantemente en la medida en que la gente actúa y reflexiona sobre sus propias propuestas de cambio traducidas en experiencias, para ajustarlas o replantearlas en función de las necesidades o dificultades identificadas. Esta constante crítica y autocrítica es protagonizada por los miembros de la comunidad, son ellos los actores que la cuestionan y la transforman. En este sentido, la comunidad investigada deja de ser únicamente objeto de investigación para convertirse en sujeto activo, capaz de modificar la realidad en la que se encuentra inmersa (Ander-Egg, 2003). Es así que la IAP propone revalorizar los saberes de las personas y su potencial transformador.

Siguiendo esta línea, hablamos de una forma de investigar capaz de democratizar el conocimiento y que está orientada a generar cambios sociales. No obstante, y con el fin de que la población adquiera capacidades investigativas en cuanto a la metodología que ha de emplearse, supone una co-implicación en el proceso de investigadores e investigadoras sociales y la población involucrada; intercambio que conlleva una fructuosa combinación de conocimientos teóricos y metodológicos con conocimientos prácticos, vivencias y experiencias de la gente. Podemos decir que existen tres elementos centrales que caracterizan este tipo de investigación: "a) el ser una metodología para el cambio; b) el fomentar la participación y autodeterminación de las personas que la utilizan, y c) ser la expresión de la relación dialéctica entre conocimiento y acción" (Durston \& Miranda, 2002, pág. 10).

Desde sus inicios la IAP aparece como un mecanismo que posibilita a la población tomar poder y encaminarse a cambios sustanciales en sus condiciones de vida. Aquí lo novedoso e inusual no es que las personas se cuestionen sobre su realidad y que persigan cambios en favor de un mayor bienestar, sino el hecho de que este cuestionamiento implique un acto investigativo, un esfuerzo intelectual y estructurado que dé respuesta a su aspiración emancipadora. La idea se origina como un medio que apunta a la construcción de una sociedad más justa y en esta medida desde sus inicios estuvo dirigida a las clases oprimidas, como una alternativa que les permita autodeterminarse en tanto se 
vuelven más críticos, creativos, propositivos y confiados de sus propias capacidades (Lewin, y otros, 1992).

En Latinoamérica surge en los años 60 como medio de planificación social y educativa, hacia finales de esta década y durante los años 70 es asumida por intelectuales de las ciencias sociales como expresión del compromiso con los movimientos populares y con las transformaciones sociopolíticas. En los años 80 aparece inmersa en un contexto donde predominaban gobiernos dictatoriales con modelos excluyentes o en medio de procesos de democratización (Durston \& Miranda, 2002). Hoy la IAP es concebida como un modelo que favorece la gobernabilidad y la integración social, partiendo de las necesidades y expectativas de la población.

Ahora bien, retomando su estrecho vínculo con la educación y, particularmente con la Educación Popular, recordar la llegada de esta propuesta a América Latina es fundamental, pues entre los años 60 y 70 confluyeron en la región una serie de disciplinas que apuntaban a un mismo fin, la toma de conciencia del pueblo sobre la complejidad de su realidad para alterarla en favor de las y los oprimidos del sistema. Entre estas disciplinas precisamente convergen la Educación Popular y la IAP, junto con otras como: la Teología de la Liberación, la Comunicación Alternativa y la Filosofía de la Liberación (Ortiz \& Borjas, 2008). Ésta, que no es una coincidencia, evidencia que ambas propuestas se encuentran alineadas, que persiguen un mismo fin.

Contraria a la noción de la educación como un acto neutral, simple transmisión de conocimientos, la Educación Popular la concibe como un acto político cargado siempre de intencionalidad que genera conciencia y politiza a la persona. Para Freire la educación verdadera es aquella que permite generar conciencia sobre las contradicciones del entorno, a lo que agrega: "Las contradicciones concienciadas no le dan más descanso, sino que vuelven insoportable la acomodación" (Freire, 2012, pág. 21). Así, una educación verdadera, resulta aquella que insta a la persona a la acción, pues la pasividad ya no le resulta posible.

Conciencia y acción son entonces dos puntales en los que la IAP y la Educación Popular empatan y que abren nuevos caminos a la persona 
en tanto se reconoce a sí misma y a su entorno, ya no como algo inalterable, sino como algo que es capaz de ser transformado. Aquella actitud inquieta que despierta la Educación Popular se ve reflejada en las ansias de conocimiento de aquel que adopta la IAP en su práctica y vida diaria. Y así como en la educación, en la IAP teoría y práctica no están disociadas, sino que dialogan como parte de un mismo proceso en el que lo teórico sirve como sustento de lo práctico (Ortiz \& Borjas, 2008).

El proceso de IAP es esencialmente dialógico, resulta de un diálogo constante entre los miembros de la comunidad y con las y los investigadores sociales que apuestan por su gente y por aquello que los mueve; así también, la Educación Popular, entendida como una "reciprocidad de conciencias" (Freire, 2012, pág. 12), se hace posible mediante una comunicación horizontal que favorece el aprendizaje mutuo entre educadores/as y educandos.

Es posible comprender, por ende, que no toda investigación coincide con la Educación Popular, pues no toda investigación es un llamado a la acción y a la transformación, no toda investigación nace de la apuesta por un mundo más justo, ni se erige sobre la base de un diálogo horizontal de quienes luchan por un mismo propósito. Investigar y educar para liberar es aquello que permite que ambas disciplinas se encuentren y fluyan en un mismo sentido.

\section{El rol de las/los educadoras/es investigadoras/es en la transformación de las prácticas educativas.}

Sin lugar a duda, los educadores y educadoras componen una de las piezas fundamentales de la esfera educativa, tal es su trascendencia que podríamos aventurarnos a afirmar que sobre ellos y ellas recae gran parte de las posibilidades de transformación de esta esfera. Contemplar la relevancia social de su práctica, su influencia en la disrupción o reproducción de lo establecido nos permite visualizar la necesidad de incidir significativamente en su formación; una formación orientada a provocar crisis permanentes, que desestabilice y promueva el ejercicio del pensamiento crítico, que cuestione constantemente, que promueva la participación activa y que crea en la necesidad de la transformación, 
en este sentido, se plantea un reto significativo en la formación de estos actores pues "formar adecuadamente al docente supone un cambio radical para transformarlo de consumidor de cursos y talleres, y repetidor de conocimientos y teorías, en productor de conocimientos y de soluciones a los problemas o situaciones problemáticas que le plantea la práctica" (Pérez, 1997).

De este modo, el educador y la educadora como sujetos productores de conocimientos, capaces de asumir su propia formacióntransformación (Pérez Esclarín, 1997), están llamados a ser investigadores de aquello que configura el sentido común de su práctica docente y que ha sido asumido como algo inalterable. Bajo el rol de educadores/ as investigadores/as tienen el reto de develar aquellos mecanismos de su práctica cotidiana que los disuaden de pensar críticamente y desencadenan prácticas tradicionales de enseñanza.

Para Paulo Freire la investigación forma parte de la naturaleza de la práctica docente, por lo tanto, a lo que debemos encaminarnos es a generar esa conciencia en los educadores y educadoras para que se perciban y asuman, por el hecho de ser educadores, como investigadores. (Freire, 2012, pág. 26). Esta mirada del docente como investigador nos invita a desmitificar la exclusividad de la producción investigativa cuya validez ha recaído únicamente en los trabajos realizados por técnicos especializados, por otro lado, esta visión nos induce a romper, a su vez, con la idea errada de que llevar a cabo investigaciones en el aula es una tarea complicada que se aleja del trabajo que el educador realiza (Kutz, 1992).

La IAP se configura como una de las técnicas de investigación en el aula que puede aportar progresivamente en esta toma de conciencia del rol del/de la docente como investigador/a. Bajo el enfoque de la IAP, los educadores/as asumen el rol de investigadores activos y autocríticos de su práctica, se asumen como aprendices de su propio trabajo, del trabajo de sus compañeros y de sus alumnos/as, de manera tal que se vuelven sujetos de conocimiento y sujetos en proceso de formación. Por otro lado, la investigación en el aula puede presentarse como una oportunidad para desarrollar y fomentar las habilidades, destrezas y cualidades de los educadores/as al proporcionarles la oportunidad de 
plantearse preguntas, observar, reflexionar, dialogar y sistematizar lo aprendido.

Podemos mirar a los procesos de IAP como espacios de autorreflexión en los que se revalorizan los saberes y experiencias adquiridas por los educadores y educadoras en su trayectoria docente. Adicionalmente, pueden convertirse en espacios en los que se comparte y promueve el sentido de responsabilidad y compromiso con la transformación social. De este modo, la investigación debe ser asumida como una práctica medular del proceso de enseñanza aprendizaje, debe constituirse en una herramienta que aporte a los educadores/as en el análisis y reflexión de sus problemáticas cotidianas, además de ser un instrumento que motive la construcción colectiva del conocimiento a partir del encuentro de los distintos saberes de los actores educativos, debe servir como medio para convocar y movilizar a la comunidad educativa hacia la transformación.

Dentro de este escenario, los educadores y las educadoras tienen un rol protagónico en la transformación, su capacidad reflexiva, autocrítica, disruptiva puede incidir significativamente en la formación de sus estudiantes y en los cambios buscados a nivel del sistema educativo.

\section{El proceso de IAP desde los centros educativos de Fe y Alegría Ecuador.}

El proceso de IAP en los centros educativos de Fe y Alegría Ecuador nace como una propuesta del CFI con miras a crear una cultura de IAP en las comunidades educativas. Partiendo de aquello que soñamos ser, un Movimiento que bajo su modelo educativo "contempla la construcción de hombres y mujeres capaces de humanizar y transformar su realidad haciendo uso de sus propias capacidades y potencialidades cognitivas y afectivas" (Cabarrús, 2003 citado en Fe y Alegría, 2016), se visualizó a este método como aquel canal que nos ayuda a ir en esa dirección. La IAP surge entonces dentro de la planificación y de los retos del CFI como una nueva posibilidad de la institución para mirarse a sí misma y como un camino para que las y los educadores cuestionen sus prácticas, su contexto y regresen sobre sus propias capacidades para transformar y transformarse. 
Es así como bajo la mirada de las y los educadores sobre lo que es y quiere ser Fe y Alegría, se inicia en el año 2017 aquello que nace como un proyecto de IAP, pero que en realidad supone la puesta en marcha de un camino que, siguiendo la línea de la Educación Popular, pretende instaurar la investigación en los centros educativos como medio para un continuo crecimiento, como una vía que apunta a la emancipación. La IAP busca, entonces, invitar a la comunidad educativa a la toma de conciencia sobre la multiplicidad de situaciones que son aún un obstáculo para alcanzar sus propósitos y un llamado a que, mediante una reflexión-acción-reflexión, entre en un ciclo que, aunque no tiene fin, implica la constante conquista de logros a pequeña, mediana y gran escala.

Como primer paso de este gran reto se impulsó un curso con un componente presencial y uno virtual, donde a través de lecturas, diálogos reflexivos, aplicación de técnicas participativas y un análisis del contexto de cada centro, las y los educadores/as investigadores/ as iniciaron un proceso de indagación en aquellas problemáticas que aquejan a su comunidad con el fin último de incidir en ellas mediante estrategias y acciones concretas que encaminen a la comunidad hacia el cambio deseado.

Este proyecto reflexivo/formativo tuvo su primer ciclo en la costa e inició uno en la sierra durante los primeros meses del 2018. En la costa los equipos de investigación durante varios meses se centraron en la elaboración de un diagnóstico participativo en torno a la problemática que identificaron como más relevante dentro de su contexto y, durante el año 2018 se ha iniciado un proceso de programación y aplicación de planes de acción construidos por los equipos, en función de dar respuesta a las necesidades expresadas en su centro. Los equipos de la sierra, cuyo proceso comenzó concretamente en febrero del año 2018, se encuentran realizando el diagnóstico participativo de su comunidad educativa.

Desde la oficina nacional de Fe y Alegría Ecuador, el CFI conformó un equipo con el fin de apoyar con herramientas y conocimientos teóricos y metodológicos las investigaciones abordadas por las educadoras y educadores. 


\section{Un espacio de catarsis ante las problemáticas de la comunidad.}

Como todo proceso pensado desde la teoría y llevado a la práctica, éste ha supuesto enfrentarse a situaciones inesperadas, algunas adversas y otras gratas sorpresas. Una de aquellas circunstancias poco previstas fue descubrir en la IAP el espacio propicio para provocar una catarsis no solo en educadores y educadoras, sino en estudiantes, madres y padres de familia. Esto sin duda nos llevó a cuestionarnos sobre cuántos y cuáles espacios de escucha cercana y activa se establecen en los centros de educación formal para atender a las múltiples preocupaciones de las personas que conforman la comunidad.

Al concebir al equipo del CFI no solamente como personas cuyo propósito en este proceso es dar seguimiento y otorgar herramientas metodológicas a los equipos, sino también como acompañantes y con esto nos referimos a personas que se preocupan por otras personas, el proceso supuso establecer cercanía y confianza entre todas y todos quienes nos hemos involucrado en el mismo.

Así, el primer y quizás el más importante paso a dar para hacer de este reto un desafío posible fue escuchar. $Y$ en esa escucha han salido a relucir problemáticas de toda índole, desde dificultades metodológicas en el aula y falta de comprensión sobre estrategias de innovación e inclusión educativa, hasta graves problemas de convivencia, inadecuados canales de diálogo entre los miembros de la comunidad educativa, fricciones entre autoridades y educadores/as, entre muchos otros. La presencia de esta diversidad de problemáticas que en ocasiones ha supuesto enfrentarse a una severa dificultad a la hora de centrarnos en una sola, permite comprender la complejidad de la esfera educativa, al menos de la educación formal tal como ha sido instaurada hasta nuestros días.

Yes que la escuela formal regida por claros parámetros instaurados ya no solamente a nivel de Estado, sino a nivel internacional, impone de una forma u otra una serie de barreras que quebrantar, implica una enorme y ardua tarea. Entre dichas barreras encontramos jerarquías que, si bien la Educación Popular y con ella Fe y Alegría, busca romper, 
siguen vigentes $y$, aunque no siempre, en múltiples ocasiones resultan ser uno de esos muros que impide que la comunicación fluya espontánea y libremente. $Y$ cuando hablamos de jerarquías, incluimos entre aquellas, unas instauradas de manera formal y otras menos formales, pero claramente asumidas por la sociedad, como la que distingue al cuerpo de educadores y educadoras del equipo directivo; la relación educandos - educadores/as; la relación de padres/madres respecto a sus hijos e hijas y hasta las divisiones que por edades están demarcadas en las aulas de los centros.

Existen también barreras físicas, que son aquellas que dividen el espacio que ocupan las y los educadores, las y los directivos, las psicólogas y psicólogos, las y los educandos, el personal administrativo $y$, por fuera, casi como un actor externo, las familias y quienes viven y trabajan en el barrio.

Desde Fe y Alegría, sin duda, uno de los retos es y ha venido siendo la integración de la comunidad educativa con todas las personas que en ella están inmersas, pero no deja de sorprender cuán distantes pueden llegar a estar y lo difícil que es migrar de una forma de ser y hacer instaurada en la cotidianidad y en el imaginario desde hace mucho tiempo atrás, a una nueva forma de concebir las relaciones que apunte a una verdadera horizontalidad.

Entonces, a la hora de entablar un diálogo, de sentarnos en mesa redonda a conversar, ya no solo desde el rol específico que desempeña cada uno, sino desde las preocupaciones que a todas y todos conciernen como invitados e invitadas a un encuentro en el que el criterio y los saberes de cada persona tiene el mismo peso, surgen, de pronto, todas aquellas preocupaciones que en el diario vivir no han tenido un espacio concreto, uno establecido para que se confronten entre sí.

Esta es la catarsis de la que hablamos. Del espacio que los encuentros del proceso de IAP ha visto necesarios establecer, donde los distintos actores de la comunidad se sientan juntos a conversar, a intercambiar percepciones, a dar ideas, a proponer, a desfogar sus frustraciones, a tomar conciencia de aquello que no habían visualizado, a escuchar de manera directa aquello que suponían. 
Y es que la ventaja de que esta metodología sea, necesariamente, participativa, presupone la escucha, la construcción colectiva de procesos y una toma de decisiones que nazca de un sentir común. Es decir, parte de la noción de construir comunidad, porque para una real transformación se precisa de muchas cabezas y de muchas manos dispuestas a alcanzar un objetivo, una meta.

El hallazgo, entonces, fue visibilizar claramente la baja presencia de canales asertivos de comunicación entre las personas que están involucradas con las escuelas y colegios de Fe y Alegría. El reto, por lo tanto, resulta aún mayor, pues partimos de que, en unos contextos, más que en otros, es necesario fortalecer la noción de comunidad, la conciencia de que somos parte de un mismo colectivo y de que solamente a través de un trabajo conjunto será posible hacer cambios profundos en las múltiples realidades que los centros educativos afrontan.

\section{La toma de conciencia sobre la complejidad del contexto}

Diríamos, entonces, que visibilizar esta ausencia de canales y espacios para una comunicación asertiva constituye un primer paso en la toma de conciencia de aquello que sucede en las comunidades educativas. La ventaja, más allá de ver esta realidad, resulta que dicha toma de conciencia vino de la mano de la escucha a posturas de diversos actores de la comunidad respecto a las situaciones conflictivas, diálogo que en sí mismo permitió una mayor comprensión de los problemas que experimentan. En algunos casos se evidenció la coincidencia de pensamientos entre varios actores, mientras que, en otros se visibilizó mayor diversidad de posturas y de puntos de vista sobre un mismo problema o sobre varios.

Aquí, por ejemplo, nos gustaría señalar un caso donde si bien el equipo de investigación había identificado como aquello que más les preocupaba dentro de su comunidad: una baja formación de las y los educadores, al ampliar la discusión a un número mayor y más diverso de personas, se esclareció que aquello que preocupaba en mayor medida eran las brechas que existen en el diálogo entre unos actores y otros, lo que refuerza lo explicado con anterioridad. El equipo 
evidenció en el debate la desconfianza presente entre miembros del cuerpo de educadores y educadoras, pero también entre estudiantes y educadores/as. Resultó clara la emergencia de acciones que acerquen a unos y otros y rompan el aislamiento presente en varias personas de la comunidad educativa por el temor a ser juzgadas y por verse inmersas en situaciones conflictivas y rumores.

Otro caso que vale destacar fue el resultado de un encuentro participativo realizado por un equipo que vio alrededor de la inclusión educativay de cómoésta se está ejecutando en su centro, la mayor dificultad que atraviesan. En el encuentro se subdividieron las y los participantes en equipos para debatir sobre los conflictos que encontraban en torno a aquella temática. Las/los participantes se agruparon de acuerdo al rol que cumplen en la institución, es decir, educadoras y educadores juntos, estudiantes por otro lado y padres y madres de familia conformaron otro equipo. De manera separada debatieron respecto a la multiplicidad de dificultades que veían en este gran ámbito. Lo curioso fue que, cuando se pidió a los equipos que identificasen de entre todas las problemáticas conversadas, cuál era aquella que consideraban central, la más preocupante, sin que hubiese una conversación entre los equipos, todos ubicaron como más significativa la baja conciencia y sensibilización de las y los miembros de la comunidad respecto a la inclusión educativa. Claro está, y en este punto vale aclarar, que dicha falta de conciencia se manifestaba de diversas formas, haciendo énfasis en distintos actores de acuerdo al equipo que lo había discutido. No obstante, se evidenciaron coincidencias en el discurso, lo que trazó el inicio del camino a recorrer por los miembros del equipo de investigación.

Por lo tanto, la reflexión partiendo de la experiencia previa de las y los miembros de la comunidad educativa y puesta en común mediante el diálogo, constituye una de las herramientas más relevantes a la hora de concienciar y esclarecer las dificultades y diversas realidades que se viven en los centros educativos.

Otra herramienta que es utilizada en ésta y otras formas de hacer investigación es una profunda indagación bibliográfica. Contrastar la realidad de la comunidad con aquellos insumos que otorgan las lecturas permitiría a quienes forman parte de ella visualizar desde 
distintas perspectivas lo que les acontece y ampliar su visión sobre el problema. Sin embargo, en esta experiencia y posiblemente en muchas otras, donde los contextos, como bien se ha remarcado no son necesariamente académicos, la profundización bibliográfica puede suponer una dificultad.

La experiencia enfocada concretamente en la lectura resultó ser muy variable de acuerdo al contexto de cada centro. $Y$ aquí hay que remarcar que, si bien Fe y Alegría ha optado desde sus inicios y hasta hoy por los sectores más vulnerables de la sociedad, refiriéndonos con esto a las condiciones socioeconómicas de sus contextos, existen importantes diferencias a la hora de situarse en la capital, en otra grande o mediana ciudad, en una pequeña población rural, en un espacio donde la atención del Estado ha estado ausente o casi ausente.

Considerando este amplio panorama, notamos que en unos espacios más que en otros, resulta más o menos necesario otorgar particular importancia al refuerzo de la práctica lectora para que ésta se convierta en un potente instrumento de investigación.

Ahora bien, cuando hablamos de un proceso de toma de conciencia que responde a una metodología concreta, donde siguiendo pasos estructurados, aunque flexibles, se induce un ejercicio de mayor criticidad y reflexión de la habitual, ésta puede suponer que quienes lo guían interpongan elementos adicionales que favorezcan una mayor comprensión de la realidad. Aquí vale recordar el rol de las y los investigadores sociales, quienes además de investigar con la población, son fuente de ciertos conocimientos teóricos y metodológicos.

Siguiendo esta línea, como parte del equipo del CFI que acompaña el proceso, hemos optado por una particular estrategia en centros educativos cuyos equipos de investigación se centraron en la indagación de problemáticas asociadas a la inequidad de género en sus contextos. Aquí, y dada la complejidad, pero también el bajo grado de conocimiento que como sociedad tenemos ante la diversidad de circunstancias y propuestas en torno a dicho tema, se propuso y abordó las investigaciones acompañadas de una formación respecto a conceptos y planteamientos teóricos. 
Así, las investigaciones en cuanto a género se dieron de la mano de un proceso paralelo de discusión y reflexión sobre la amplitud de este campo y sobre la realidad concreta de Ecuador en torno al mismo. Los centros educativos que trabajan en ello son aquellos que, a través de una indagación del análisis de su contexto, demostraron tener los índices más altos de violencia de género. Aquí cabe hacer un paréntesis en cuanto a la relevancia de esta problemática que, si bien no solamente está presente en el ámbito educativo, es preciso realzar el rol determinante que tiene éste en su progresiva transformación. Según un estudio del año 2014 realizado desde el Consejo Nacional para la Igualdad de Género, en Ecuador el 60,6\% de mujeres han experimentado algún tipo de violencia de género y aquella ha sido propiciada mayormente por su pareja o expareja. Datos que indican que: el $87,3 \%$ de la violencia física, el $76,3 \%$ de la violencia psicológica, el $53,5 \%$ de la violencia sexual y el $61 \%$ de la violencia patrimonial son perpetradas por parejas o exparejas de las víctimas, resultan alarmantes.

De este modo, antes de adentrarnos de lleno en las investigaciones, se destinó varios encuentros a diálogos acompañados de lecturas, ejercicios y exposición a material audiovisual que permitiera ahondar en dicha disciplina previo al análisis de las problemáticas más relevantes identificadas en sus instituciones educativas.

La experiencia en estos equipos demostró una clara evolución en cuanto a la comprensión inicial y la alcanzada a medida que fue avanzando el proceso. Es de resaltar, que una vez iniciadas las investigaciones, se siguió ahondando en algunas problemáticas generales asociadas a género, mediante espacios de debate y diálogo al inicio de los encuentros destinados a acompañar y a dar seguimiento a las investigaciones. Esto como un componente adicional al tratamiento concreto de los problemas que cada equipo seleccionó.

Los encuentros participativos con estos equipos supusieron también una dinámica particular. $Y$ vale destacarlos frente a lo vivido con otros equipos, justamente por la complejidad y por la dificultad antes señalada en torno al tema. Los encuentros con otros miembros de la comunidad educativa implicaron también la exposición a material audiovisual y a información sobre lo que es y lo que gira alrededor de 
la inequidad de género. Así las y los participantes dialogaron sobre la información recibida previo al conversatorio con las y los integrantes de los equipos de investigación. Los aportes de los participantes enriquecieron, al igual que en otros casos, de manera significativa los hallazgos y la comprensión de los problemas concretos que están siendo indagados. Sin embargo, lo que llama la atención en estos casos es que resulta un tema del que poco o nada se conversa en la mayoría de familias y dentro de los centros en su rutina habitual. Eso supuso que además de un intercambio de experiencias y percepciones, se manifestara un cierto grado de sorpresa ante algunos puntos y una introspección en los participantes que en otros contextos difícilmente hubiese tenido lugar.

Vemos entonces, que la toma de conciencia en medio de un proceso de IAP, si bien es algo que de manera natural ocurre dadas las condiciones que este método propicia o debe propiciar, el incluir actividades no necesariamente contempladas como parte del proceso, en aquello casos en los que se considere pertinente, puede aportar en gran medida a una reflexión más profunda y, por ende, a conclusiones más valiosas en los diagnósticos que los equipos realicen dentro de su contexto.

\section{El curso de IAP como primer paso para encaminarnos hacia una cultura de investigación acción.}

Habiendo reflexionado ya sobre la importancia de este método en la educación y su contribución en la toma de conciencia colectiva y desarrollo del pensamiento crítico-reflexivo, quizás nos surgen preguntas como: ¿cómo iniciar procesos de IAP en los centros educativos?, ¿de qué manera garantizar la participación activa de los educadores y las educadoras en estos procesos?, ¿cómo procurar una formación para educadores y educadoras que promueva la visión del docente como investigador?, ¿de qué modo garantizar espacios de diálogo y reflexión dentro de la dinámica de los centros educativos?, ¿cómo promover espacios de encuentro entre los diferentes actores de la comunidad educativa? Estas interrogantes han sido el punto de partida del proceso de investigación iniciado, y continúan planteándonos una serie de retos a ser asumidos. 
Partiendo de este llamado a emprender procesos de IAP, nos vimos en la necesidad de trazar algunas estrategias concretas para impulsar y dinamizar proyectos de investigación bajo esta metodología en las comunidades educativas. Al respecto, nos gustaría compartir las acciones concretas consideradas en algunos centros de Fe y Alegría Ecuador.

\section{Un acercamiento al curso virtual de IAP}

Con seguridad, existe una variedad de caminos para promover procesos de investigación-acción protagonizados por educadores y educadoras, desde el CFI hemos visualizado, como estrategia, iniciar la ejecución de proyectos de investigación a partir de un curso virtual con aval académico reforzado con jornadas presenciales. Se ha concebido el curso de IAP como una oferta formativa que apuesta por la construcción colectiva de conocimientos desde una mirada crítica y reflexiva. Adicionalmente, esta estrategia se ha consolidado como un mecanismo efectivo para motivar la participación de las educadoras y educadores y constituye el primer medio para captar su interés.

El curso virtual de IAP, además de ser una propuesta formativa, ha facilitado el seguimiento del desarrollo de los proyectos de investigación planteados por los equipos, de manera que, las actividades programadas han respondido a las distintas fases de la investigación-acción:

\section{Tabla 1: Fases del proyecto de IAP}

\section{Diagnóstico participativo:}

- Detección de las principales problemáticas.

- Apertura a las percepciones y puntos de vista de los actores de la comunidad educativa.

- Delimitación de la problemática investigada.

- Planteamiento de los objetivos de la investigación.

- Acercamiento a la literatura (Revisión de fuentes bibliográficas).

- Elaboración y aplicación de técnicas de investigación participativas.

- Organización y análisis de la información recolectada en el trabajo de campo.

- Sistematización de la experiencia de Investigación Acción Participativa. 


\section{Plan de Acción.}

- Construcción colectiva del Plan de Acción.

- Ejecución de las propuestas concretas.

- Evaluación y sistematización.

\section{Detección de nuevos problemas y /o dificultades.}

Fuente: Elaboración propia.

Dentro de la IAP las fases de investigación expuestas previamente adquieren sentido en la medida en que son efectuadas dentro de una espiral de reflexión colectiva permanente, esta particularidad de la IAP devela las limitaciones de concentrar el proceso únicamente en la virtualidad, en consecuencia, el componente presencial se convierte en un elemento fundamental del proceso, y de cierto modo, podría afirmarse que la persistencia de los educadores y educadoras en el proceso se ha visto mediada por éste. Entonces, es necesario resaltar la importancia de los encuentros, así como los espacios de reflexión establecidos con otros actores de la comunidad educativa, ambos forman parte de la metodología propuesta en el curso y se han encaminado a la generación de una cultura de diálogo reflexivo en los centros educativos.

Como se ha señalado con anterioridad, uno de los componentes trascendentales de la IAP es la acción, al respecto, es importante resaltar que, bajo este enfoque, la investigación pierde su valor si no se encuentra orientada a la acción, sabiendo que se concibe a este proceso como un ciclo que no acaba en la implementación de las propuestas, sino que supone una contante revisión crítica de lo realizado.

Podemos definir al plan de acción como la invitación a construir colectivamente las transformaciones que soñamos, es así como, "pensar un plan implica sentarnos juntos a imaginar qué queremos, hacia dónde vamos, con qué contamos, en qué tiempo y lugar lo vamos a hacer, con qué posibilidades contamos y qué alianzas tenemos que hacer" (Gyssels, 2011, pág. 14). Además, en él se materializa la conciencia renovada de los educadores/a y los insta a desarrollar su creatividad, por ende, es un reflejo del compromiso asumido por los/as educadores/ as con la transformación. 
Bajo estas premisas, el proyecto de IAP ha considerado como punto indispensable la construcción y puesta en práctica de planes de acción diseñados por los equipos. Actualmente, esta fase ha iniciado en el ciclo costa, para ella hemos previsto que la principal motivación sea el compromiso de transformar las comunidades educativas a las que pertenecen los educadores/as investigadores/as, por tal razón, esta etapa no se estructura bajo la modalidad de curso virtual con aval académico, sino que supuso la participación voluntaria de los equipos que desearan continuar en ella. Cabe resaltar el interés de gran parte de los/as educadores/as por continuar en el proceso, lo que refleja su motivación por lograr cambios dentro de sus contextos.

En definitiva, podemos decir que el curso de IAP ha sido un instrumento para dar apertura a la investigación dentro de los centros educativos, en este sentido, lo concebimos como el primer paso para encaminarnos hacia una cultura de investigación. Sin embargo, es conveniente resaltar que éste, por sí solo, no logra el fin último perseguido: lograr que los/as docentes se asuman como educadores/ as investigadores/as, y que en consecuencia promuevan procesos de IAP como parte de su accionar educativo. Será necesario continuar reflexionando sobre la importancia de la investigación en la práctica cotidiana, generar esta conciencia implica un trabajo permanente que debe abordarse siguiendo otras dinámicas.

\section{Conclusiones}

Vemos entonces que a través de la IAP es posible empezar a articularnos, pues resulta un medio para el encuentro e intercambio de saberes y experiencias. El proceso, que como bien lo hemos dicho, es apenas el inicio de aquello a lo que nos proyectamos, ha permitido en primera instancia aproximarnos como equipo de investigadoras a las demandas de las y los educadores y a las diversas realidades que se viven en los centros. Ha posibilitado visibilizar la inmensa labor que realizan los educadores/as, así como sus dificultades, incertidumbres, los pequeños logros que van conquistando en su día a día y sus aspiraciones profesionales y personales.

Trabajar con ellas y ellos ha supuesto el desafío de contextualizar el proceso y de confrontar nuestra visión del educador con su propia forma de concebirse, entendiendo que su rol no se limita a la escolaridad, 
sino a una formación en y para la vida. Ha significado, a su vez, mirarnos a nosotras mismas, en un esfuerzo por entendernos como educadoras y en esa medida asumir nuestro rol de personas llamadas a construir la sociedad.

La experiencia de IAP desde nuestra perspectiva ha implicado una constante apertura al cambio, a replantearnos continuamente la ruta a seguir para darle sentido al proceso; en esta medida, comprendemos que la propuesta continúa en construcción, pues son los educadores y educadoras de los centros quienes están llamados/as a reinventarla. Bajo esta premisa, nos enfrentamos al desafío de persistir animadas/os ante el ideal de alcanzar verdaderas transformaciones. Nos proyectamos a que educadores y educadoras encuentren una motivación en el proceso de IAP, que siempre será un camino inacabado. Por lo tanto, el reto está en mantenernos en movimiento, en no dejar que el proceso iniciado quede archivado, sino volverlo parte de nuestra práctica cotidiana. La educación soñada será siempre un horizonte, y por ende demanda de nosotros/as un ejercicio permanente de deconstrucción y construcción creativa que nos mantenga caminando hacia aquello que queremos ser.

\section{Bibliografía}

Ander-Egg, E. (2003). Repensando la Investigación-Acción-Participativa. Lumen Humanitas.

Camacho, G. (2014). La violencia de género contra las mujeres en el Ecuador: Análisis de los resultados de la Encuesta Nacional sobre Relaciones Familiares y Violencia de Género contra las Mujeres. Quito: Consejo Nacional para la Igualdad de Genero.

Corrales, O., \& Jimenez, M. d. (s.f.). El docente como investigador. Revista Educacion, 73-79.

Durston, J., \& Miranda, F. (2002). Experiencias y metodología de la investigación participativa. Santiago de Chile: CEPAL.

Fe y Alegría. (2016). Horizonte Pedagógico Pastoral Fe y Alegría Ecuador. Quito.

Freire, P. (2012). Pedagogía del oprimido. Madrid: Siglo XXI de España Editores.

Gyssels, S. (2011). Planificar para el cambio. Bogotá.

Kutz, C. (1992). Teacher Research: Myths and Realities. 
Lewin, K., Stavenhagen, R., Fals Borda, O., Zamosc, L., Kemmis, S., \& Rahman, A. (1992). La investigación-acción participativa: Inicios y desarrollo. Madrid: Editorial Popular .

Ortiz, M., \& Borjas, B. (2008). La Investigación Acción Participativa: aporte de Fals Borda a la educación popular. Espacio Abierto, 615-627.

Pérez, A. (1997). Más y mejor educación para todos. Maracaibo. 